\title{
Numerical design of thorium and uranium fuel samples irradiation in lead environment
}

\author{
Mikolaj Oettingen \\ AGH University of Science and Technology, Faculty of Energy and Fuels, Department of Nuclear Energy, al. Mickiewicza 30, \\ 30-059 Krakow, Poland
}

Received: 11 February 2020 / Received in final form: 14 April 2020 / Accepted: 9 July 2020

\begin{abstract}
The paper shows capabilities of thorium-lead fuel assembly for design of irradiation experiments on $\mathrm{ThO}_{2}$ and natural $\mathrm{UO}_{2}$ fuel samples using radioisotope neutron source. The main purpose of the current analysis was to determine the irradiation environment in the samples, especially: neutron spectrum, power, activity, reaction rates, production of ${ }^{233} \mathrm{~Pa}$ and ${ }^{239} \mathrm{~Np}$ as well as breeding of ${ }^{233} \mathrm{U}$ and ${ }^{239} \mathrm{Pu}$. An advanced threedimensional numerical model for Monte Carlo radiation transport and burnup simulations was developed using the Monte Carlo Continuous Energy Burnup Code (MCB). The versatility of the assembly gives a perfect opportunity to perform many irradiation experiments for R\&D on the thorium and uranium fuel cycle in a different material and geometrical environments.
\end{abstract}

\section{Introduction}

The paper presents the neutronic analysis of the irradiation experiments planned at the AGH University of Science and Technology in Krakow, Poland, using a state-of-the-art thorium-lead (Th-Pb) fuel assembly. The experiments consider the irradiation of $\mathrm{ThO}_{2}$ and natural $\mathrm{UO}_{2}$ fuel samples using radioisotope $\mathrm{Cf}$ neutron source. The fuel sample is embedded in the fuel rod placed in the array of the remaining 236 rods of either thorium or natural uranium, reflected by lead rods and bricks. The flexibility of the $\mathrm{Th}-\mathrm{Pb}$ assembly allows to perform many irradiation experiments with different geometrical configurations of the core. The purpose of the current analysis is to determine the irradiation environment in the sample, especially: neutron spectrum, power, activity, production of ${ }^{233} \mathrm{~Pa}$ and ${ }^{239} \mathrm{~Np}$ as well as breeding of ${ }^{233} \mathrm{U}$ and ${ }^{239} \mathrm{Pu}$ for further comparison with the results of the irradiation experiments. An advanced threedimensional numerical model for Monte Carlo radiation transport and burnup simulations was developed using the Monte Carlo Continuous Energy Burnup Code (MCB) [1], developed at the AGH University. The code was equipped with ENDF/B-VII.1 [2] and JEFF3.1 [3] nuclear data libraries for comparative study. The analysis was performed for fuel samples containing $\mathrm{ThO}_{2}$ and natural $\mathrm{UO}_{2}$ for the comparison of breeding capabilities of ${ }^{233} \mathrm{U}$ and ${ }^{239} \mathrm{Pu}$.

The research mainly focuses on the $\mathrm{ThO}_{2}$ fuel sample, which was chosen because of an increasing interest in the

\footnotetext{
* e-mail: moettin@agh.edu.pl
}

thorium fuel cycle and a demand for more basic research into thorium fuels $[4,5]$. In general, thorium seems to be an attractive option for nuclear fuel mainly due to its abundance, reduced need for enrichment in the fuel cycle, high conversion ratios to ${ }^{233} \mathrm{U}$ in a thermal neutron spectrum, low production of plutonium and minor actinides and high proliferation resistance. Although many investigations have been carried out into thorium-based fuels while thorium-fuelled power reactors have operated in the past, the thorium fuel cycle as a whole has never been fully developed. As yet, there is no commercial fabrication or reprocessing infrastructure for the thorium fuel cycle, unlike the vast available infrastructure for the uranium fuel cycle. Nevertheless, the experience gained on thorium-based fuels in test and power reactors as well as the results of the numerical analysis are very encouraging [6-9]. Therefore, the implementation of thorium fuels to the power reactor must be further supported by scientific research to allow better understanding of all components of the thorium fuel cycle. In spite of the large amount of available neutronic data for numerical modelling, better neutronic characteristics supported by experimental validation with dedicated infrastructure such as the $\mathrm{Th}-\mathrm{Pb}$ assembly are needed.

In Section 2, the technical specification of the Th- $\mathrm{Pb}$ assembly and the developed numerical model for the Monte Carlo modelling are described. The results of the numerical modelling are shown in Section 3. Section 4 contains a summary of the study and discussion about possible improvements in the applied methodology. 


\section{Thorium-lead assembly numerical model}

Figure 1 shows the Th- $\mathrm{Pb}$ fuel assembly located in the shielded irradiation bunker in the radiometric laboratory of the AGH University. The thorium core lays on the steel bottom support plate. The upper cylindrical support plate and aluminium grid fasten the core from the top. In addition, four side bars located symmetrically around the core stabilize the assembly. The side reflector surrounding the core was fabricated using lead bricks with dimensions $100 \mathrm{~mm} \times 100 \mathrm{~mm} \times 50 \mathrm{~mm}$ and hexagonal lead rods with the length of $120 \mathrm{~cm}$. The cylindrical $\mathrm{ThO}_{2}$ and $\mathrm{UO}_{2}$ rods have the same length of $120 \mathrm{~cm}$ and radius of $5.32 \mathrm{~mm}$. The

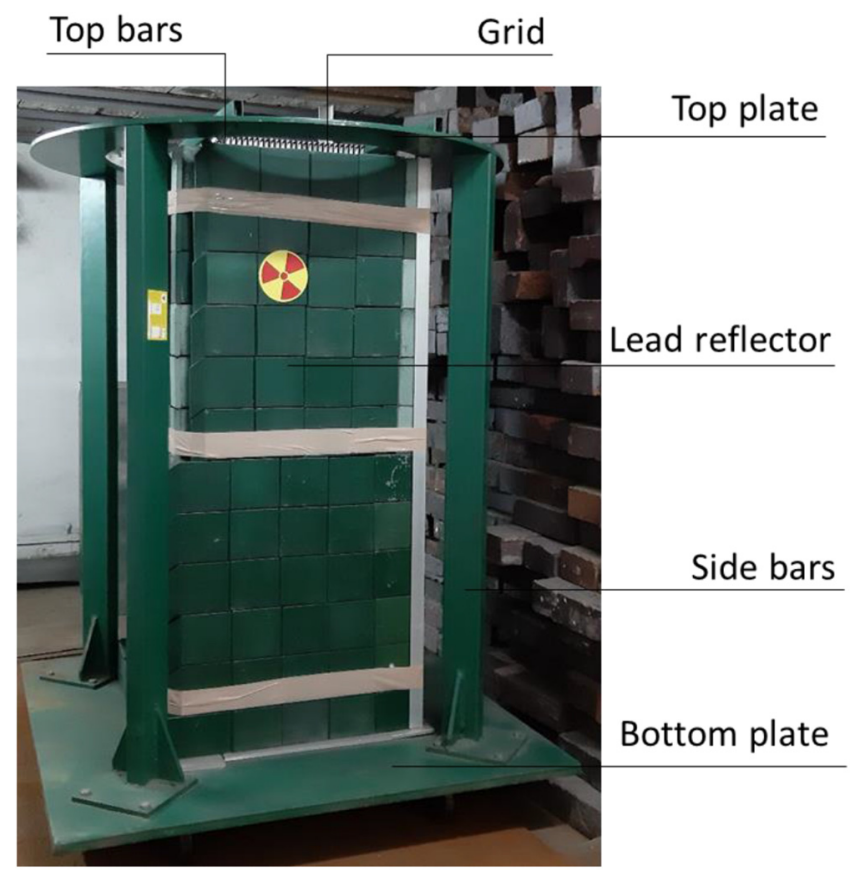

Fig. 1. Th- $\mathrm{Pb}$ fuel assembly in the irradiation bunker. active high, gap and clad thickness in both cases equals 100 , 0.012 and $0.085 \mathrm{~cm}$ respectively. The pitch of hexagonal assembly grid is $1.27 \mathrm{~cm}$. The free space of $0.04 \mathrm{~cm}$ between rods was designed to facilitate subtraction of the chosen rods without dismantling of the whole assembly. This, in turn, facilitates introduction of the new elements like e.g. radiation detectors, samples or other materials comprising experiments. In general, Th- $\mathrm{Pb}$ assembly is characterised by a quite simple construction, which is an advantage in its maintenance during planning, execution and post-processing of irradiation experiments.

The numerical reconstruction of the assembly was based on the performed profilometric measurements as well as specification of the fuel rods provided by the supplier (Bhabha Atomic Research Centre, India) [10]. The active height of the fertile material $(1000 \mathrm{~mm})$ was numerically divided into four radial (RZ) and three axial (AZ) zones presented in Figures 2 and 3. The subsequent zone of $25 \mathrm{~mm}$ represents the fuel sample, which was additionally divided into five similar axial segments for the estimation of axial distributions of key physical parameters, such as activity, power and production of the investigated isotopes. The height of each segment equals $0.5 \mathrm{~cm}$, which gives the volume of $0.436 \mathrm{~cm}^{3}$. The segment mass depends on the fuel type; in the case of $\mathrm{ThO}_{2}$, it is $4.06 \mathrm{~g}$ and in the case of $\mathrm{UO}_{2}-$ $4.54 \mathrm{~g}$. The isotopic composition of uranium and thorium dioxide is shown in Tables 1 and 2 respectively. For better numerical precision, results of the simulation were scored in six symmetric zones representing the samples around the neutron source, as it is shown in Figure 2. Then, the results were averaged for particular segments. The Th rods are surrounded by the hexagonal matrix of $\mathrm{Pb}$ rods and $\mathrm{Pb}$ bricks respectively. The production of ${ }^{233} \mathrm{U}$ and ${ }^{239} \mathrm{Pu}$ is the most effective in the thermal neutron spectrum due to the high cross-section for neutron capture on ${ }^{232} \mathrm{Th}$ and ${ }^{238} \mathrm{U}$. Therefore, for the planned irradiation experiments, thermalisation zone made of hexagonal polyethylene rods $\left(\mathrm{C}_{2} \mathrm{H}_{4}\right)$ was introduced to the assembly. For the current study, the neutron source was surrounded by two layers of

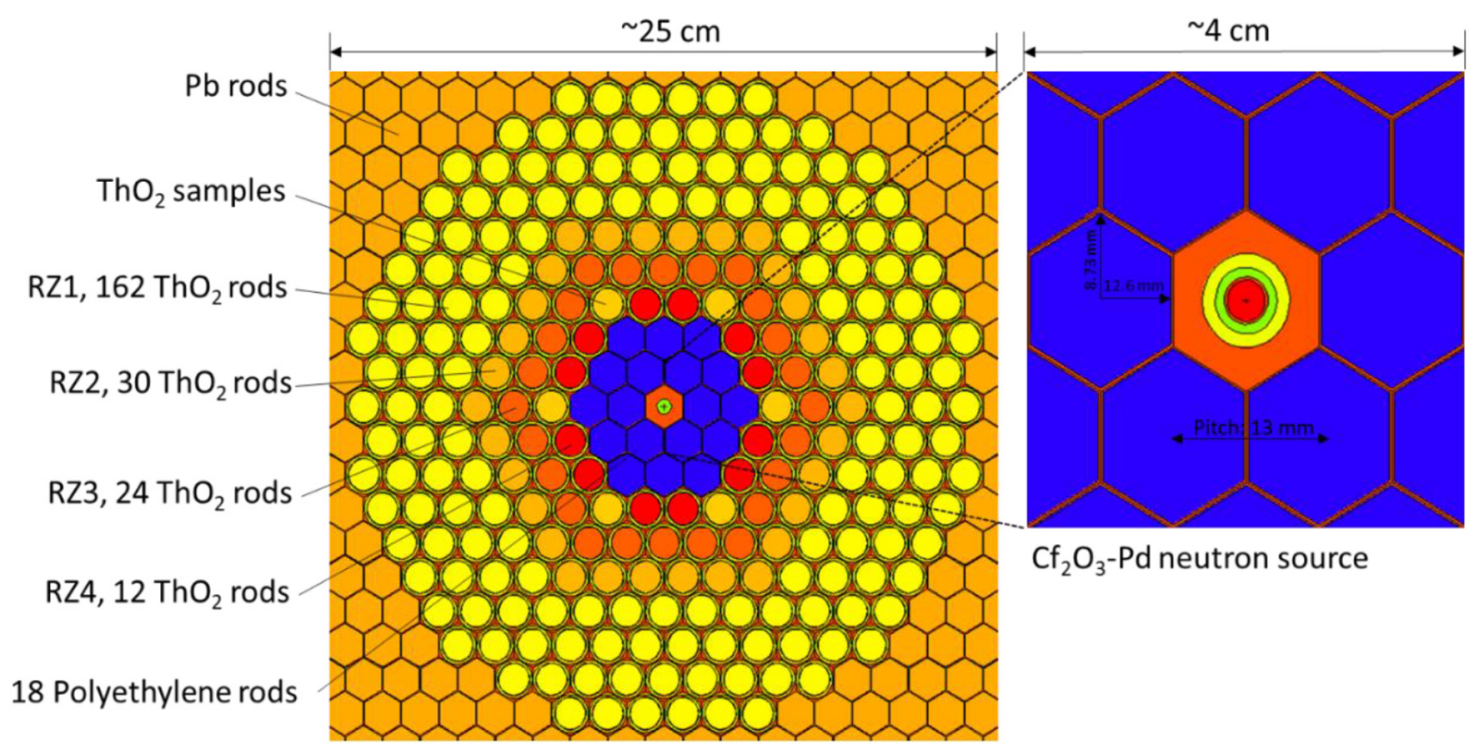

Fig. 2. Radial crosscut of the assembly's numerical model and the neutron source. 


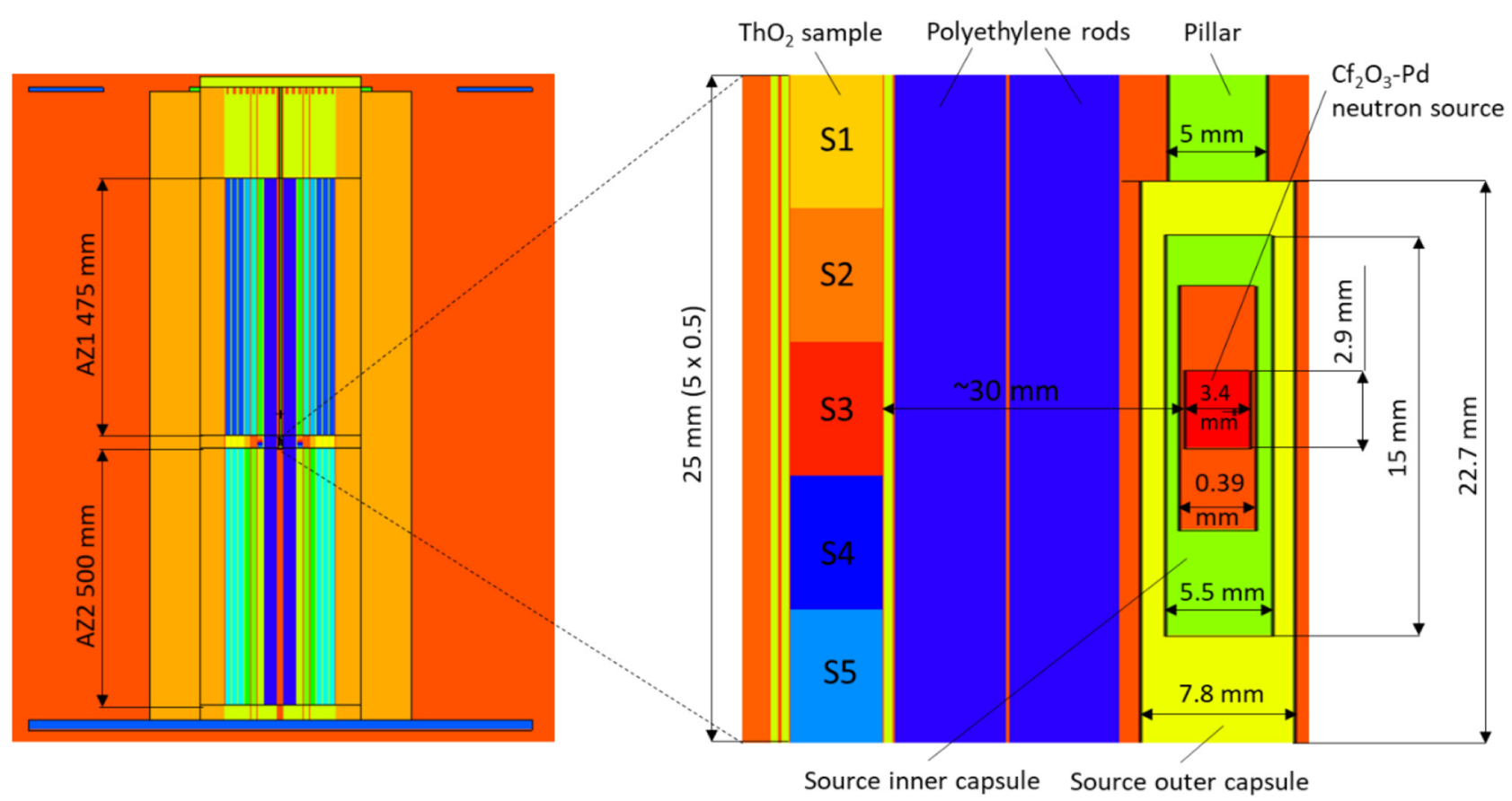

Fig. 3. Axial crosscut of the assembly's numerical model and the neutron source.

Table 1. The isotopic composition of the thorium sample.

\begin{tabular}{llll}
\hline \multicolumn{3}{c}{$\mathrm{ThO}_{2}$} \\
\hline No & Isotope & at. [\%] & Segment mass [g] \\
\hline 1 & ${ }^{232} \mathrm{Th}$ & $3.33 \mathrm{E}-01$ & $3.56 \mathrm{E}+00$ \\
2 & ${ }^{230} \mathrm{Th}$ & $1.33 \mathrm{E}-04$ & $8.46 \mathrm{E}-03$ \\
3 & $\mathrm{Th}$ & $3.33 \mathrm{E}-01$ & $3.57 \mathrm{E}+00$ \\
4 & ${ }^{16} \mathrm{O}$ & $6.67 \mathrm{E}-01$ & $4.91 \mathrm{E}-01$ \\
\hline
\end{tabular}

Table 2. The isotopic composition of the uranium sample.

\begin{tabular}{llll}
\hline & \multicolumn{3}{c}{$\mathrm{UO}_{2}$} \\
\hline No & Isotope & at. [\%] & Segment mass [g] \\
\hline 1 & ${ }^{234} \mathrm{U}$ & $1.93 \mathrm{E}-05$ & $2.28 \mathrm{E}-04$ \\
2 & ${ }^{235} \mathrm{U}$ & $2.43 \mathrm{E}-03$ & $2.88 \mathrm{E}-02$ \\
3 & ${ }^{238} \mathrm{U}$ & $3.31 \mathrm{E}-01$ & $3.97 \mathrm{E}+00$ \\
4 & $\mathrm{U}$ & $3.33 \mathrm{E}-01$ & $4.00 \mathrm{E}+00$ \\
5 & ${ }^{16} \mathrm{O}$ & $6.67 \mathrm{E}-01$ & $5.38 \mathrm{E}-01$ \\
\hline
\end{tabular}

polyethylene rods of 6 and 12 rods respectively. As a result, the total number of $\mathrm{ThO}_{2} / \mathrm{UO}_{2}$ rods in the assembly for the planned experiment equals 234, which gives $\mathrm{ThO}_{2}$ the mass of $192 \mathrm{~kg}$, including $169 \mathrm{~kg}$ of $\mathrm{Th}$, and $\mathrm{UO}_{2}-$ the mass of $214 \mathrm{~kg}$, including $189 \mathrm{~kg}$ of $\mathrm{U}\left(1.3 \mathrm{~kg}{ }^{235} \mathrm{U}, 187.5 \mathrm{~kg}{ }^{238} \mathrm{U}\right.$ and trace amount of $\left.{ }^{234} \mathrm{U}\right)$. The materials used for the numerical modelling and their densities are shown in Table 3. For the modelling of the grid, side bars and top bars, which are characterized by the complicated geometry the volumetric homogenisation technique was applied. The neutron source was modelled according to the specification of the manufacturer [11] as a $\mathrm{Cf}_{2} \mathrm{O}_{3}-\mathrm{Pd}$ cermet wire closed in two stainless steel capsules. Figure 2 shows the location of the source in the middle of the assembly. The chosen source has intensity of $10^{8} \mathrm{n} / \mathrm{s}$, which gives mass of $43 \mu \mathrm{g}$ of ${ }^{252} \mathrm{Cf}$. The planned irradiation time equals 25 days, but it can be easily shortened or extended, depends on experiment specification.

\section{Results}

Section 3 presents results of the numerical modelling using the Monte Carlo Continuous Energy Burnup Code (MCB) for neutron transport and burnup simulation $[1,12]$. The calculations were performed for the two sets of nuclear data libraries i.e. ENDF/B-VII.1 and JEFF3.1 for comparative analysis. The supercomputer Prometheus of the Academic Computer Centre of AGH University was used for numerical simulations. The numerical precision of the obtained results is much below $0.5 \%$. In the following subsections the characteristic of neutron spectra in the samples, mass evolutions of crucial isotopes $\left({ }^{233} \mathrm{~Pa},{ }^{239} \mathrm{~Np}\right.$, ${ }^{233} \mathrm{U},{ }^{239} \mathrm{Pu}$ ) as well as power and activity evolutions are shown.

\subsection{Neutron spectrum}

Figure 4 shows the absolute neutron spectrum, while Figure 5 presents the relative neutron spectrum calculated in 100 energy group structures for ENDF/B-VII.1 nuclear data libraries at the beginning of irradiation. The shape of the spectrum is similar for both applied libraries and for particular time steps, as it is mainly formed by the $\mathrm{Cf}$ 
Table 3. Materials used in the numerical modelling.

\begin{tabular}{llll}
\hline No & Element & Material & Density $\left[\mathrm{g} / \mathrm{cm}^{3}\right]$ \\
\hline 1 & Fuel pellets & $\mathrm{ThO}_{2},{ }^{\text {nat }} \mathrm{UO}_{2}$ & $9.3,10.4$ \\
2 & Neutron source & $\mathrm{Cf}_{2} \mathrm{O}_{3}-\mathrm{Pd}$ & 12.023 \\
3 & Reflector & $\mathrm{Pb}$ & 11.35 \\
4 & Rods & Polyethylene & 0.92 \\
5 & Source capsules, pillar & SS304L Steel & 8 \\
6 & Fixing bars, plugs, clad & Aluminium & 2.7 \\
7 & Plates & Steel & 7.85 \\
8 & Free space & Air & 0.001205 \\
9 & Grid & Steel $52 \%+$ air $48 \%$ & 4.08 \\
10 & Side bars & Steel $31 \%+$ air $69 \%$ & 2.4 \\
11 & Top bars & Steel $13 \%+$ air $87 \%$ & 1.01 \\
\hline
\end{tabular}

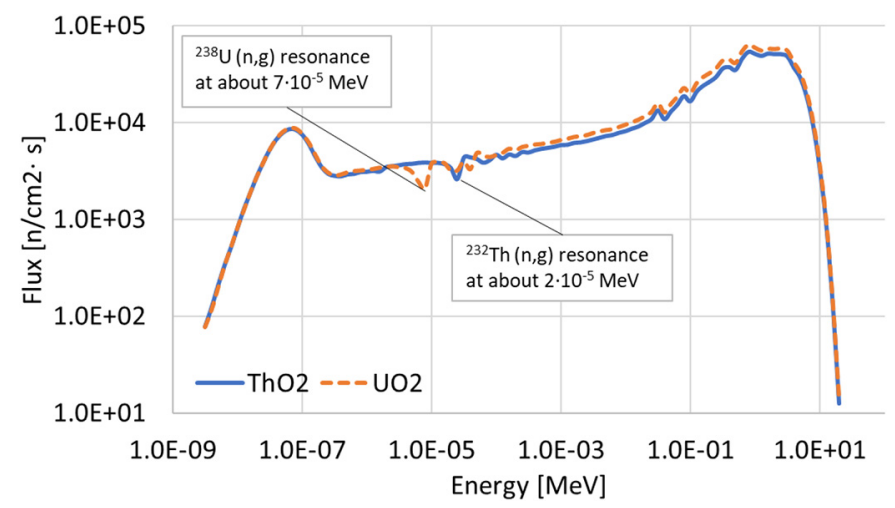

Fig. 4. Absolute neutron spectrum for ENDF/B-VII.1 in the central fuel segment.

neutron source and polyethylene thermalisation zones. The isotopic changes in 25 days of irradiation and the $\mathrm{Cf}$ decay do not influence it significantly. The differences between JEFF3.1 and ENDF/B-VII.1 libraries depend on the energy group but mostly they are below $1 \%$ on average, and below $0.25 \%$ for both samples. In the spectrum, two major peaks related to thermal and fast neutrons are presented. The main difference between the $\mathrm{ThO}_{2}$ and $\mathrm{UO}_{2}$ fuel samples comes from the flux depressions due to the radiative capture cross-sections on ${ }^{232} \mathrm{Th}$ and ${ }^{238} \mathrm{U}$. The first large resonance for ${ }^{232} \mathrm{Th}$ appears at about $2 \times 10^{-5}$ $\mathrm{MeV}$ and for ${ }^{238} \mathrm{U}-$ at about $7 \times 10^{-5} \mathrm{MeV}$ in the epithermal energy range, which is visible in Figure 4. For ${ }^{238} \mathrm{U}$, two following flux depressions due to resonances at about $2 \times 10^{-5}$ and $4 \times 10^{-5} \mathrm{MeV}$ are visible. The spectrum for $\mathrm{UO}_{2}$ is harder, with $17.40 \%$ of neutrons below $1 \mathrm{keV}$ in the thermal and epithermal energy range, $7.28 \%(>1 \mathrm{eV})$ and $10.13 \%(>1 \mathrm{eV} \bigwedge \leq 1 \mathrm{keV})$ respectively. In the case of $\mathrm{ThO}_{2}, 19.39 \%$ neutrons are in the thermal and epithermal energy range, $8.13 \%$ and $11.26 \%$ respectively. The reason for the difference are the higher effective macroscopic neutron absorption reaction rates for uranium $\left({ }^{234} \mathrm{U}+{ }^{235} \mathrm{U}\right.$ $+{ }^{238} \mathrm{U}: 2.58 \mathrm{E}+04\left[1 / \mathrm{s} \cdot \mathrm{cm}^{3}\right]$ for ENDF/B-VII.1) compared with thorium $\left({ }^{230} \mathrm{Th}+{ }^{232} \mathrm{Th}\right.$ : $1.76 \mathrm{E}+04\left[1 / \mathrm{s} \cdot \mathrm{cm}^{3}\right]$ for ENDF/B-VII.1), see Tables 5 and 7. In general, the absolute neutron flux is higher in the case of the $\mathrm{UO}_{2}$ fuel

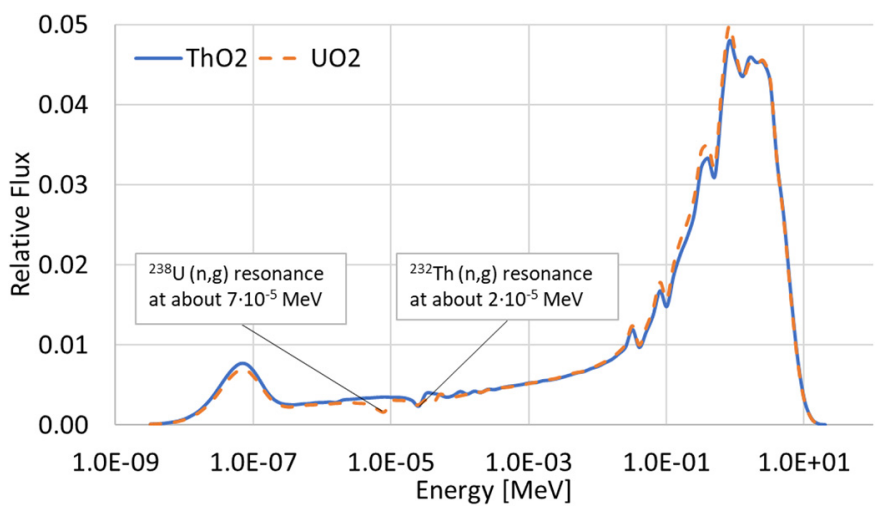

Fig. 5. Relative neutron spectrum for ENDF/B-VII.1 in the central fuel segment.

sample $\left(1.27 \mathrm{E}+06 \mathrm{n} / \mathrm{cm}^{2} \cdot \mathrm{s}\right)$ compared with the $\mathrm{ThO}_{2}$ fuel sample $\left(1.11 \mathrm{E}+06 \mathrm{n} / \mathrm{cm}^{2} \cdot \mathrm{s}\right)$ due to the presence of fissionable ${ }^{235} \mathrm{U}$ in the fresh fuel composition and the surplus fissions in the thermal and epithermal energy range.

\subsection{Mass evolution}

The mass evolution of ${ }^{233} \mathrm{~Pa}$ and ${ }^{233} \mathrm{U}$ for both nuclear data libraries and the sample segments is shown in Figures 6 and 7. ${ }^{233} \mathrm{~Pa}$ increases almost linearly during the irradiation time of 25 days, as it is formed shortly after the neutron absorption on ${ }^{232} \mathrm{Th}$ from decay of ${ }^{233} \mathrm{Th}$ with a halflifetime of $22.3 \mathrm{~min}$. The formation of ${ }^{233} \mathrm{~Pa}$ depends on the neutron flux and thus on the power of the segments. The highest number of atoms for ENDF/B-VII.1 at the end of irradiation was obtained in the central segment and the lowest - in the peripheral segments, $1.16 \times 10^{10}$ and $1.13 \times 10^{10}$ respectively. The results for JEFF3.1 nuclear data libraries show the higher concentration of ${ }^{233} \mathrm{~Pa}$ of about $2 \%$ for the whole irradiation cycle. The rising concentration of ${ }^{233} \mathrm{~Pa}$ shows that the equilibrium state was not achieved during irradiation. The concentration of ${ }^{233} \mathrm{U}$ increases significantly during the irradiation time because of its breeding from ${ }^{232} \mathrm{Th}$. At the end of irradiation, it equals $4.10 \times 10^{9}$ atoms for ENDF/B-VII.1 nuclear data 
Table 4. Final concentrations of the investigated isotopes.

\begin{tabular}{|c|c|c|c|c|}
\hline \multicolumn{5}{|c|}{ ENDF/B-VII.1 [atoms] } \\
\hline Segment & ${ }^{233} \mathrm{~Pa}$ & ${ }^{233} \mathrm{U}$ & ${ }^{239} \mathrm{~Np}$ & ${ }^{239} \mathrm{Pu}$ \\
\hline 1 & $1.13 \mathrm{E}+10$ & $4.00 \mathrm{E}+09$ & $1.76 \mathrm{E}+09$ & $1.11 \mathrm{E}+10$ \\
\hline 2 & $1.15 \mathrm{E}+10$ & $4.06 \mathrm{E}+09$ & $1.79 \mathrm{E}+09$ & $1.13 \mathrm{E}+10$ \\
\hline 3 & $1.16 \mathrm{E}+10$ & $4.10 \mathrm{E}+09$ & $1.80 \mathrm{E}+09$ & $1.13 \mathrm{E}+10$ \\
\hline 4 & $1.15 \mathrm{E}+10$ & $4.07 \mathrm{E}+09$ & $1.80 \mathrm{E}+09$ & $1.13 \mathrm{E}+10$ \\
\hline 5 & $1.13 \mathrm{E}+10$ & $3.99 \mathrm{E}+09$ & $1.76 \mathrm{E}+09$ & $1.10 \mathrm{E}+10$ \\
\hline Total & $5.72 \mathrm{E}+10$ & $2.02 \mathrm{E}+10$ & $8.91 \mathrm{E}+09$ & $5.60 \mathrm{E}+10$ \\
\hline \multicolumn{5}{|c|}{ JEFF3.1 [atoms] } \\
\hline Segment & ${ }^{233} \mathrm{~Pa}$ & ${ }^{233} \mathrm{U}$ & ${ }^{239} \mathrm{~Np}$ & ${ }^{239} 239 \mathrm{Pu}$ \\
\hline 1 & $1.15 \mathrm{E}+10$ & $4.08 \mathrm{E}+09$ & $1.77 \mathrm{E}+09$ & $1.11 \mathrm{E}+10$ \\
\hline 2 & $1.18 \mathrm{E}+10$ & $4.16 \mathrm{E}+09$ & $1.81 \mathrm{E}+09$ & $1.13 \mathrm{E}+10$ \\
\hline 3 & $1.18 \mathrm{E}+10$ & $4.18 \mathrm{E}+09$ & $1.81 \mathrm{E}+09$ & $1.14 \mathrm{E}+10$ \\
\hline 4 & $1.18 \mathrm{E}+10$ & $4.16 \mathrm{E}+09$ & $1.80 \mathrm{E}+09$ & $1.13 \mathrm{E}+10$ \\
\hline 5 & $1.15 \mathrm{E}+10$ & $4.07 \mathrm{E}+09$ & $1.77 \mathrm{E}+09$ & $1.11 \mathrm{E}+10$ \\
\hline Total & $5.84 \mathrm{E}+10$ & $2.06 \mathrm{E}+10$ & $8.95 \mathrm{E}+09$ & $5.63 \mathrm{E}+10$ \\
\hline
\end{tabular}

Table 5. Microscopic and macroscopic reaction rates at the beginning of irradiation for ${ }^{230} \mathrm{Th}$ and ${ }^{232} \mathrm{Th}$.

\begin{tabular}{|c|c|c|c|c|c|c|c|c|c|}
\hline \multicolumn{5}{|c|}{${ }^{230} \mathrm{Th}-\mathrm{ENDF} / \mathrm{B}-\mathrm{VII} .1$} & \multicolumn{5}{|c|}{${ }^{230} \mathrm{Th}-\mathrm{JEFF} 3.1$} \\
\hline \multirow[t]{2}{*}{ Segment } & \multicolumn{2}{|c|}{$r_{\mathrm{eff}}[1 / \mathrm{s}]$} & \multicolumn{2}{|c|}{$R_{\text {eff }}\left[1 / \mathrm{s} \cdot \mathrm{cm}^{3}\right]$} & \multirow[t]{2}{*}{ Segment } & \multicolumn{2}{|c|}{$r_{\text {eff }}[1 / \mathrm{s}]$} & \multicolumn{2}{|c|}{$R_{\text {eff }}\left[1 / \mathrm{s} \cdot \mathrm{cm}^{3}\right]$} \\
\hline & $(n, g)$ & $(\mathrm{n}, \mathrm{f})$ & $(\mathrm{n}, \mathrm{g})$ & $(n, f)$ & & $(\mathrm{n}, \mathrm{g})$ & $(\mathrm{n}, \mathrm{f})$ & $(\mathrm{n}, \mathrm{g})$ & $(\mathrm{n}, \mathrm{f})$ \\
\hline 1 & $1.52 \mathrm{E}-09$ & $9.58 \mathrm{E}-12$ & $1.30 \mathrm{E}+02$ & $8.10 \mathrm{E}-01$ & 1 & $1.24 \mathrm{E}-09$ & $8.38 \mathrm{E}-12$ & $1.05 \mathrm{E}+02$ & $7.09 \mathrm{E}-01$ \\
\hline 2 & $1.53 \mathrm{E}-09$ & $1.01 \mathrm{E}-11$ & $1.31 \mathrm{E}+02$ & $8.54 \mathrm{E}-01$ & 2 & $1.26 \mathrm{E}-09$ & $8.85 \mathrm{E}-12$ & $1.07 \mathrm{E}+02$ & $7.49 \mathrm{E}-01$ \\
\hline 3 & $1.54 \mathrm{E}-09$ & $1.03 \mathrm{E}-11$ & $1.31 \mathrm{E}+02$ & $8.72 \mathrm{E}-01$ & 3 & $1.28 \mathrm{E}-09$ & $9.02 \mathrm{E}-12$ & $1.08 \mathrm{E}+02$ & $7.63 \mathrm{E}-01$ \\
\hline 4 & $1.53 \mathrm{E}-09$ & $1.01 \mathrm{E}-11$ & $1.31 \mathrm{E}+02$ & $8.53 \mathrm{E}-01$ & 4 & $1.26 \mathrm{E}-09$ & $8.83 \mathrm{E}-12$ & $1.06 \mathrm{E}+02$ & $7.47 \mathrm{E}-01$ \\
\hline 5 & $1.52 \mathrm{E}-09$ & $9.58 \mathrm{E}-12$ & $1.30 \mathrm{E}+02$ & $8.11 \mathrm{E}-01$ & 5 & $1.25 \mathrm{E}-09$ & $8.38 \mathrm{E}-12$ & $1.05 \mathrm{E}+02$ & $7.09 \mathrm{E}-01$ \\
\hline & ${ }^{232} \mathrm{Tl}$ & $-\mathrm{ENDF} / 1$ & -VII.1 & & & & Th - JEFH & & \\
\hline
\end{tabular}

\begin{tabular}{lllllllllll}
\hline \multirow{2}{*}{ Segment } & \multicolumn{2}{c}{$r_{\text {eff }}[1 / \mathrm{s}]$} & \multicolumn{2}{c}{$R_{\text {eff }}[1 / \mathrm{s} \cdot \mathrm{cm} 3]$} & \multicolumn{2}{c}{ Segment } & \multicolumn{2}{c}{$r_{\text {eff }}[1 / \mathrm{s}]$} & \multicolumn{2}{c}{$R_{\text {eff }}[1 / \mathrm{s} \cdot \mathrm{cm} 3]$} \\
\hline & $(\mathrm{n}, \mathrm{g})$ & $(\mathrm{n}, \mathrm{f})$ & $(\mathrm{n}, \mathrm{g})$ & $(\mathrm{n}, \mathrm{f})$ & & $(\mathrm{n}, \mathrm{g})$ & $(\mathrm{n}, \mathrm{f})$ & $(\mathrm{n}, \mathrm{g})$ & $(\mathrm{n}, \mathrm{f})$ \\
\hline 1 & $7.68 \mathrm{E}-19$ & $3.44 \mathrm{E}-20$ & $1.63 \mathrm{E}+04$ & $7.29 \mathrm{E}+02$ & 1 & & $7.85 \mathrm{E}-19$ & $3.56 \mathrm{E}-20$ & $1.66 \mathrm{E}+04$ & $7.56 \mathrm{E}+02$ \\
2 & $7.82 \mathrm{E}-19$ & $3.64 \mathrm{E}-20$ & $1.66 \mathrm{E}+04$ & $7.71 \mathrm{E}+02$ & 2 & & $7.98 \mathrm{E}-19$ & $3.77 \mathrm{E}-20$ & $1.69 \mathrm{E}+04$ & $7.99 \mathrm{E}+02$ \\
3 & $7.88 \mathrm{E}-19$ & $3.71 \mathrm{E}-20$ & $1.67 \mathrm{E}+04$ & $7.86 \mathrm{E}+02$ & 3 & & $8.04 \mathrm{E}-19$ & $3.84 \mathrm{E}-20$ & $1.71 \mathrm{E}+04$ & $8.15 \mathrm{E}+02$ \\
4 & $7.84 \mathrm{E}-19$ & $3.63 \mathrm{E}-20$ & $1.66 \mathrm{E}+04$ & $7.69 \mathrm{E}+02$ & 4 & & $8.01 \mathrm{E}-19$ & $3.76 \mathrm{E}-20$ & $1.70 \mathrm{E}+04$ & $7.98 \mathrm{E}+02$ \\
5 & $7.68 \mathrm{E}-19$ & $3.44 \mathrm{E}-20$ & $1.63 \mathrm{E}+04$ & $7.30 \mathrm{E}+02$ & 5 & & $7.83 \mathrm{E}-19$ & $3.57 \mathrm{E}-20$ & $1.66 \mathrm{E}+04$ & $7.56 \mathrm{E}+02$ \\
\hline
\end{tabular}

libraries in the central segment, which is consistent with the concentration of ${ }^{233} \mathrm{~Pa}$. The results for JEFF3.1 nuclear data libraries present ${ }^{233} \mathrm{U}$ concentrations higher by about $2 \%$. The axial distribution of ${ }^{233} \mathrm{~Pa}$ and ${ }^{233} \mathrm{U}$ corresponds to the power distribution. The concentration of ${ }^{233} \mathrm{U}$ will still increase after the end of irradiation because of the ${ }^{233} \mathrm{~Pa}$ decay.

The concentration of ${ }^{239} \mathrm{~Np}$ during the irradiation time reaches the equilibrium state at about 15 days, which is shown in Figure 8. This is caused by the equilibrium between ${ }^{239} \mathrm{~Np}$ formation from decay of ${ }^{239} \mathrm{U}$ and its further decay to ${ }^{239} \mathrm{Pu}$. The axial distribution of ${ }^{239} \mathrm{~Np}$ corresponds to the power distribution. At the end of irradiation for ENDF/B-VII.1 nuclear data libraries, the highest concen- tration of ${ }^{239} \mathrm{~Np}$ was obtained in the central segment $\left(1.80 \times 10^{9}\right.$ atoms $)$ and the lowest concentration - in the peripheral segments $\left(1.76 \times 10^{9}\right.$ atoms $)$. The calculations with JEFF3.1 nuclear data libraries show the concentration of ${ }^{239} \mathrm{~Np}$ higher by about $1 \%$. The concentration of ${ }^{239} \mathrm{Pu}$ increases with the irradiation time due to its breeding from ${ }^{238} \mathrm{U}$, which is shown in Figure 9. The highest concentration of $1.14 \times 10^{10}$ atoms corresponds to the central segment of the sample and the lowest concentration of $1.11 \times 10^{10}$ atoms - to the peripheral segment of the sample. JEFF3.1 nuclear data libraries in general show the concentration of ${ }^{239} \mathrm{Pu}$ higher by about $1 \%$. The calculations also show some minor differences between the concentrations in the symmetric segments (1-5 and 
Table 6. Microscopic and macroscopic reaction rates at the end of irradiation for ${ }^{233} \mathrm{U}$ and ${ }^{233} \mathrm{~Pa}$.

\begin{tabular}{|c|c|c|c|c|c|c|c|c|c|}
\hline \multicolumn{5}{|c|}{${ }^{233} \mathrm{U}-\mathrm{ENDF} / \mathrm{B}-\mathrm{VII} .1$} & \multicolumn{5}{|c|}{${ }^{233} \mathrm{U}-\mathrm{JEFF} 3.1$} \\
\hline \multirow[t]{2}{*}{ Segment } & \multicolumn{2}{|c|}{$r_{\text {eff }}[1 / \mathrm{s}]$} & \multicolumn{2}{|c|}{$R_{\text {eff }}[1 / \mathrm{s} \cdot \mathrm{cm} 3]$} & \multirow[t]{2}{*}{ Segment } & \multicolumn{2}{|c|}{$r_{\text {eff }}[1 / \mathrm{s}]$} & \multicolumn{2}{|c|}{$R_{\mathrm{eff}}[1 / \mathrm{s} \cdot \mathrm{cm} 3]$} \\
\hline & $(\mathrm{n}, \mathrm{g})$ & $(\mathrm{n}, \mathrm{f})$ & $(n, g)$ & $(\mathrm{n}, \mathrm{f})$ & & $(n, g)$ & $(n, f)$ & $(n, g)$ & $(\mathrm{n}, \mathrm{f})$ \\
\hline 1 & $4.99 \mathrm{E}-18$ & $4.28 \mathrm{E}-17$ & $4.57 \mathrm{E}-08$ & $3.92 \mathrm{E}-07$ & 1 & $4.87 \mathrm{E}-18$ & $4.25 \mathrm{E}-17$ & $4.56 \mathrm{E}-08$ & $3.98 \mathrm{E}-07$ \\
\hline 2 & $5.06 \mathrm{E}-18$ & $4.34 \mathrm{E}-17$ & $4.71 \mathrm{E}-08$ & $4.04 \mathrm{E}-07$ & 2 & $4.94 \mathrm{E}-18$ & $4.32 \mathrm{E}-17$ & $4.71 \mathrm{E}-08$ & $4.12 \mathrm{E}-07$ \\
\hline 3 & $5.07 \mathrm{E}-18$ & $4.35 \mathrm{E}-17$ & $4.76 \mathrm{E}-08$ & $4.09 \mathrm{E}-07$ & 3 & $4.96 \mathrm{E}-18$ & $4.34 \mathrm{E}-17$ & $4.76 \mathrm{E}-08$ & $4.16 \mathrm{E}-07$ \\
\hline 4 & $5.05 \mathrm{E}-18$ & $4.34 \mathrm{E}-17$ & $4.71 \mathrm{E}-08$ & $4.05 \mathrm{E}-07$ & 4 & $4.94 \mathrm{E}-18$ & $4.32 \mathrm{E}-17$ & $4.71 \mathrm{E}-08$ & $4.12 \mathrm{E}-07$ \\
\hline 5 & $4.99 \mathrm{E}-18$ & $4.29 \mathrm{E}-17$ & $4.57 \mathrm{E}-08$ & $3.93 \mathrm{E}-07$ & 5 & $4.86 \mathrm{E}-18$ & $4.25 \mathrm{E}-17$ & $4.53 \mathrm{E}-08$ & $3.96 \mathrm{E}-07$ \\
\hline \multicolumn{5}{|c|}{${ }^{233} \mathrm{~Pa}-\mathrm{ENDF} / \mathrm{B}-\mathrm{VII} .1$} & \multicolumn{5}{|c|}{${ }^{233} \mathrm{~Pa}-$ JEFF3.1 } \\
\hline Segment & \multicolumn{2}{|c|}{$r e f f[1 / \mathrm{s}]$} & \multicolumn{2}{|c|}{$\operatorname{Reff}[1 / \mathrm{s} \cdot \mathrm{cm} 3]$} & Segment & \multicolumn{2}{|c|}{ reff $[1 / \mathrm{s}]$} & \multicolumn{2}{|c|}{$R$ eff $[1 / \mathrm{s} \cdot \mathrm{cm} 3]$} \\
\hline & $(\mathrm{n}, \mathrm{g})$ & $(\mathrm{n}, \mathrm{f})$ & $(\mathrm{n}, \mathrm{g})$ & $(\mathrm{n}, \mathrm{f})$ & & $(\mathrm{n}, \mathrm{g})$ & $(n, f)$ & $(\mathrm{n}, \mathrm{g})$ & $(\mathrm{n}, \mathrm{f})$ \\
\hline 1 & $1.04 \mathrm{E}-17$ & $1.08 \mathrm{E}-19$ & $2.70 \mathrm{E}-07$ & $2.79 \mathrm{E}-09$ & 1 & $1.03 \mathrm{E}-17$ & $2.09 \mathrm{E}-19$ & $2.73 \mathrm{E}-07$ & $5.55 \mathrm{E}-09$ \\
\hline 2 & $1.06 \mathrm{E}-17$ & $1.14 \mathrm{E}-19$ & $2.80 \mathrm{E}-07$ & $2.99 \mathrm{E}-09$ & 2 & $1.06 \mathrm{E}-17$ & $2.21 \mathrm{E}-19$ & $2.84 \mathrm{E}-07$ & $5.97 \mathrm{E}-09$ \\
\hline 3 & $1.06 \mathrm{E}-17$ & $1.16 \mathrm{E}-19$ & $2.83 \mathrm{E}-07$ & $3.09 \mathrm{E}-09$ & 3 & $1.06 \mathrm{E}-17$ & $2.26 \mathrm{E}-19$ & $2.87 \mathrm{E}-07$ & $6.13 \mathrm{E}-09$ \\
\hline 4 & $1.06 \mathrm{E}-17$ & $1.13 \mathrm{E}-19$ & $2.79 \mathrm{E}-07$ & $2.99 \mathrm{E}-09$ & 4 & $1.06 \mathrm{E}-17$ & $2.21 \mathrm{E}-19$ & $2.86 \mathrm{E}-07$ & $5.96 \mathrm{E}-09$ \\
\hline 5 & $1.04 \mathrm{E}-17$ & $1.08 \mathrm{E}-19$ & $2.70 \mathrm{E}-07$ & $2.79 \mathrm{E}-09$ & 5 & $1.03 \mathrm{E}-17$ & $2.09 \mathrm{E}-19$ & $2.72 \mathrm{E}-07$ & $5.53 \mathrm{E}-09$ \\
\hline
\end{tabular}

Table 7. Microscopic and macroscopic reaction rates at the end of irradiation for ${ }^{234} \mathrm{U},{ }^{235} \mathrm{U}$ and ${ }^{238} \mathrm{U}$.

\begin{tabular}{|c|c|c|c|c|c|c|c|c|c|}
\hline \multicolumn{5}{|c|}{${ }^{234} \mathrm{U}-\mathrm{ENDF} / \mathrm{B}-\mathrm{VII} .1$} & \multicolumn{5}{|c|}{${ }^{234} \mathrm{U}-\mathrm{JEFF} 3.1$} \\
\hline \multirow[t]{2}{*}{ Segment } & \multicolumn{2}{|c|}{$r_{\text {eff }}[1 / \mathrm{s}]$} & \multicolumn{2}{|c|}{$R_{\text {eff }}\left[1 / \mathrm{s} \cdot \mathrm{cm}^{3}\right]$} & \multirow[t]{2}{*}{ Segment } & \multicolumn{2}{|c|}{$r_{\text {eff }}[1 / \mathrm{s}]$} & \multicolumn{2}{|c|}{$R_{\text {eff }}\left[1 / \mathrm{s} \cdot \mathrm{cm}^{3}\right]$} \\
\hline & $(n, g)$ & $(\mathrm{n}, \mathrm{f})$ & $(\mathrm{n}, \mathrm{g})$ & $(\mathrm{n}, \mathrm{f})$ & & $(n, g)$ & $(\mathrm{n}, \mathrm{f})$ & $(\mathrm{n}, \mathrm{g})$ & $(\mathrm{n}, \mathrm{f})$ \\
\hline 1 & $1.35 \mathrm{E}-17$ & $7.39 \mathrm{E}-19$ & $1.84 \mathrm{E}+01$ & $9.95 \mathrm{E}-01$ & 1 & $1.38 \mathrm{E}-17$ & $7.36 \mathrm{E}-19$ & $1.86 \mathrm{E}+01$ & $9.91 \mathrm{E}-01$ \\
\hline 2 & $1.39 \mathrm{E}-17$ & $7.72 \mathrm{E}-19$ & $1.87 \mathrm{E}+01$ & $1.04 \mathrm{E}+00$ & 2 & $1.40 \mathrm{E}-17$ & $7.69 \mathrm{E}-19$ & $1.88 \mathrm{E}+01$ & $1.04 \mathrm{E}+00$ \\
\hline 3 & $1.40 \mathrm{E}-17$ & $7.85 \mathrm{E}-19$ & $1.88 \mathrm{E}+01$ & $1.06 \mathrm{E}+00$ & 3 & $1.43 \mathrm{E}-17$ & $7.82 \mathrm{E}-19$ & $1.92 \mathrm{E}+01$ & $1.05 \mathrm{E}+00$ \\
\hline 4 & $1.39 \mathrm{E}-17$ & $7.71 \mathrm{E}-19$ & $1.87 \mathrm{E}+01$ & $1.04 \mathrm{E}+00$ & 4 & $1.41 \mathrm{E}-17$ & $7.69 \mathrm{E}-19$ & $1.90 \mathrm{E}+01$ & $1.04 \mathrm{E}+00$ \\
\hline \multicolumn{6}{|c|}{${ }^{235} \mathrm{U}-$ ENDF/B-VII.1 } & \multicolumn{4}{|c|}{${ }^{235} \mathrm{U}-\mathrm{JEFF} 3.1$} \\
\hline Segment & \multicolumn{2}{|c|}{$r_{\text {eff }}[1 / \mathrm{s}]$} & \multicolumn{2}{|c|}{$R_{\text {eff }}[1 / \mathrm{s} \cdot \mathrm{cm} 3]$} & Segment & \multicolumn{2}{|c|}{$r_{\text {eff }}[1 / \mathrm{s}]$} & \multicolumn{2}{|c|}{$R_{\text {eff }}[1 / \mathrm{s} \cdot \mathrm{cm} 3]$} \\
\hline & $(\mathrm{n}, \mathrm{g})$ & $(\mathrm{n}, \mathrm{f})$ & $(\mathrm{n}, \mathrm{g})$ & $(n, f)$ & & $(n, g)$ & $(\mathrm{n}, \mathrm{f})$ & $(n, g)$ & $(\mathrm{n}, \mathrm{f})$ \\
\hline 1 & $7.77 \mathrm{E}-18$ & $3.71 \mathrm{E}-17$ & $1.32 \mathrm{E}+03$ & $6.28 \mathrm{E}+03$ & 1 & $7.83 \mathrm{E}-18$ & $3.75 \mathrm{E}-17$ & $1.33 \mathrm{E}+03$ & $6.35 \mathrm{E}+03$ \\
\hline 2 & $7.88 \mathrm{E}-18$ & $3.76 \mathrm{E}$ & $1.34 \mathrm{E}+03$ & 6.37 & 2 & $7.93 \mathrm{E}-18$ & $3.79 \mathrm{E}-$ & $1.34 \mathrm{E}+03$ & $6.42 \mathrm{E}+03$ \\
\hline 3 & $7.91 \mathrm{E}-18$ & $3.77 \mathrm{E}-17$ & $1.34 \mathrm{E}+03$ & $6.39 \mathrm{E}+03$ & 3 & $7.95 \mathrm{E}-18$ & $3.80 \mathrm{E}-17$ & $1.35 \mathrm{E}+03$ & $6.44 \mathrm{E}+03$ \\
\hline 4 & $7.89 \mathrm{E}-18$ & $3.76 \mathrm{E}-17$ & $1.34 \mathrm{E}+03$ & $6.38 \mathrm{E}+03$ & 4 & $7.93 \mathrm{E}-18$ & $3.79 \mathrm{E}-17$ & $1.34 \mathrm{E}+03$ & $6.43 \mathrm{E}+0$ \\
\hline 5 & $7.78 \mathrm{E}-18$ & $3.72 \mathrm{E}-17$ & $1.32 \mathrm{E}+03$ & $6.30 \mathrm{E}+03$ & 5 & $7.83 \mathrm{E}-18$ & $3.74 \mathrm{E}-17$ & $1.33 \mathrm{E}+03$ & $6.34 \mathrm{E}+0$ \\
\hline \multicolumn{5}{|c|}{${ }^{238} \mathrm{U}-\mathrm{ENDF} / \mathrm{B}-\mathrm{VII} .1$} & \multicolumn{5}{|c|}{${ }^{238} \mathrm{U}-\mathrm{JEFF} 3.1$} \\
\hline Segment & \multicolumn{2}{|c|}{$r_{\text {eff }}[1 / \mathrm{s}]$} & \multicolumn{2}{|c|}{$R_{\text {eff }}[1 / \mathrm{s} \cdot \mathrm{cm} 3]$} & Segment & \multicolumn{2}{|c|}{$r_{\text {eff }}[1 / \mathrm{s}]$} & \multicolumn{2}{|c|}{$R_{\text {eff }}[1 / \mathrm{s} \cdot \mathrm{cm} 3]$} \\
\hline & $(\mathrm{n}, \mathrm{g})$ & $(n, f)$ & $(\mathrm{n}, \mathrm{g})$ & $(\mathrm{n}, \mathrm{f})$ & & $(\mathrm{n}, \mathrm{g})$ & $(\mathrm{n}, \mathrm{f})$ & $(\mathrm{n}, \mathrm{g})$ & $(\mathrm{n}, \mathrm{f})$ \\
\hline 1 & $5.95 \mathrm{E}-19$ & $1.59 \mathrm{E}-19$ & $1.37 \mathrm{E}+04$ & $3.66 \mathrm{E}+03$ & 1 & $6.00 \mathrm{E}-19$ & $1.57 \mathrm{E}-19$ & $1.38 \mathrm{E}+04$ & $3.63 \mathrm{E}+0$ \\
\hline 2 & $6.08 \mathrm{E}-19$ & $1.67 \mathrm{E}-19$ & $1.40 \mathrm{E}+04$ & $3.85 \mathrm{E}+03$ & 2 & $6.10 \mathrm{E}-19$ & $1.66 \mathrm{E}-19$ & $1.41 \mathrm{E}+04$ & $3.82 \mathrm{E}+03$ \\
\hline 3 & $6.13 \mathrm{E}-19$ & $1.70 \mathrm{E}-19$ & $1.41 \mathrm{E}+04$ & $3.91 \mathrm{E}+03$ & 3 & $6.14 \mathrm{E}-19$ & $1.68 \mathrm{E}-19$ & $1.42 \mathrm{E}+04$ & $3.88 \mathrm{E}+03$ \\
\hline 4 & $6.09 \mathrm{E}-19$ & $1.67 \mathrm{E}-19$ & $1.40 \mathrm{E}+04$ & $3.84 \mathrm{E}+03$ & 4 & $6.12 \mathrm{E}-19$ & $1.65 \mathrm{E}-19$ & $1.41 \mathrm{E}+04$ & $3.81 \mathrm{E}+0$ \\
\hline 5 & $5.96 \mathrm{E}-19$ & $1.59 \mathrm{E}-19$ & $1.37 \mathrm{E}+04$ & $3.66 \mathrm{E}+03$ & 5 & $6.01 \mathrm{E}-19$ & $1.57 \mathrm{E}-19$ & $1.39 \mathrm{E}+04$ & $3.63 \mathrm{E}+0$ \\
\hline
\end{tabular}

2-4) of the samples. The difference for all isotopes and time steps is below $0.4 \%$ and it is attributed to the stochastic approach of the Monte Carlo method. The total final concentrations for the investigated isotopes in the sample are shown in Table 4.
In addition, Tables $5,6,7$ and 8 present the effective microscopic $r_{\text {eff }}(1 / \mathrm{s})$ and macroscopic $R_{\text {eff }}\left(1 / \mathrm{s} \cdot \mathrm{cm}^{3}\right)$ reaction rates for the sample segments. The reaction rates for the isotopes present in the initial fuel composition $\left.{ }^{230} \mathrm{Th},{ }^{232} \mathrm{Th},{ }^{234} \mathrm{U},{ }^{235} \mathrm{U},{ }^{238} \mathrm{U}\right)$ were calculated at the 
Table 8. Microscopic and macroscopic reaction rates at the end of irradiation for ${ }^{239} \mathrm{Pu}$ and ${ }^{239} \mathrm{~Np}$.

\begin{tabular}{|c|c|c|c|c|c|c|c|c|c|}
\hline \multicolumn{5}{|c|}{${ }^{239} \mathrm{Pu}-\mathrm{ENDF} / \mathrm{B}-\mathrm{VII} .1$} & \multicolumn{5}{|c|}{${ }^{239} \mathrm{Pu}-\mathrm{JEFF} 3.1$} \\
\hline \multirow[t]{2}{*}{ Segment } & \multicolumn{2}{|c|}{ reff $[1 / \mathrm{s}]$} & \multicolumn{2}{|c|}{$R_{\text {eff }}[1 / \mathrm{s} \cdot \mathrm{cm} 3]$} & \multirow[t]{2}{*}{ Segment } & \multicolumn{2}{|c|}{$r_{\text {eff }}[1 / \mathrm{s}]$} & \multicolumn{2}{|c|}{$\operatorname{Reff}[1 / \mathrm{s} \cdot \mathrm{cm} 3]$} \\
\hline & $(n, g)$ & $(n, f)$ & $(\mathrm{n}, \mathrm{g})$ & $(\mathrm{n}, \mathrm{f})$ & & $(n, g)$ & $(n, f)$ & $(\mathrm{n}, \mathrm{g})$ & $(\mathrm{n}, \mathrm{f})$ \\
\hline 1 & $3.30 \mathrm{E}-17$ & $6.73 \mathrm{E}-17$ & $8.38 \mathrm{E}-07$ & $1.71 \mathrm{E}-06$ & 1 & $3.33 \mathrm{E}-17$ & $6.80 \mathrm{E}-17$ & $8.52 \mathrm{E}-07$ & $1.74 \mathrm{E}-06$ \\
\hline 2 & $3.34 \mathrm{E}-17$ & $6.82 \mathrm{E}-17$ & $8.63 \mathrm{E}-07$ & $1.76 \mathrm{E}-06$ & 2 & $3.37 \mathrm{E}-17$ & $6.88 \mathrm{E}-17$ & $8.76 \mathrm{E}-07$ & $1.79 \mathrm{E}-06$ \\
\hline 3 & $3.35 \mathrm{E}-17$ & $6.84 \mathrm{E}-17$ & $8.72 \mathrm{E}-07$ & $1.78 \mathrm{E}-06$ & 3 & $3.39 \mathrm{E}-17$ & $6.91 \mathrm{E}-17$ & $8.84 \mathrm{E}-07$ & $1.80 \mathrm{E}-06$ \\
\hline 4 & $3.34 \mathrm{E}-17$ & $6.83 \mathrm{E}-17$ & $8.65 \mathrm{E}-07$ & $1.77 \mathrm{E}-06$ & 4 & $3.37 \mathrm{E}-17$ & $6.88 \mathrm{E}-17$ & $8.76 \mathrm{E}-07$ & $1.79 \mathrm{E}-06$ \\
\hline & $3.30 \mathrm{E}-17$ & $6.74 \mathrm{E}-17$ & $8.35 \mathrm{E}-07$ & $1.70 \mathrm{E}-06$ & 5 & $3.33 \mathrm{E}-17$ & $6.80 \mathrm{E}-17$ & $8.50 \mathrm{E}-07$ & $1.73 \mathrm{E}-06$ \\
\hline & \multicolumn{4}{|c|}{${ }^{239} \mathrm{~Np}-\mathrm{ENDF} / \mathrm{B}-\mathrm{VII} .1$} & \multicolumn{5}{|c|}{${ }^{239} \mathrm{~Np}-\mathrm{JEFF} 3.1$} \\
\hline Segment & \multicolumn{2}{|c|}{$r_{\text {eff }}[1 / \mathrm{s}]$} & \multicolumn{2}{|c|}{$R_{\text {eff }}[1 / \mathrm{s} \cdot \mathrm{cm} 3]$} & Segment & \multicolumn{2}{|c|}{$r_{\text {eff }}[1 / \mathrm{s}]$} & \multicolumn{2}{|c|}{$\operatorname{Reff}[1 / \mathrm{s} \cdot \mathrm{cm} 3]$} \\
\hline & $(\mathrm{n}, \mathrm{g})$ & $(\mathrm{n}, \mathrm{f})$ & $(\mathrm{n}, \mathrm{g})$ & $(\mathrm{n}, \mathrm{f})$ & & $(n, g)$ & $(\mathrm{n}, \mathrm{f})$ & $(n, g)$ & $(n, f)$ \\
\hline 1 & $1.78 \mathrm{E}-17$ & $3.13 \mathrm{E}-19$ & $7.17 \mathrm{E}-08$ & $1.27 \mathrm{E}-09$ & 1 & $1.22 \mathrm{E}-17$ & $9.36 \mathrm{E}-19$ & $4.94 \mathrm{E}-08$ & $3.80 \mathrm{E}-09$ \\
\hline 2 & $1.81 \mathrm{E}-17$ & $3.29 \mathrm{E}-19$ & $7.41 \mathrm{E}-08$ & $1.35 \mathrm{E}-09$ & 2 & $1.24 \mathrm{E}-17$ & $9.78 \mathrm{E}-19$ & $5.11 \mathrm{E}-08$ & $4.05 \mathrm{E}-09$ \\
\hline 3 & $1.81 \mathrm{E}-17$ & $3.35 \mathrm{E}-19$ & $7.47 \mathrm{E}-08$ & $1.38 \mathrm{E}-09$ & 3 & $1.24 \mathrm{E}-17$ & $9.93 \mathrm{E}-19$ & $5.16 \mathrm{E}-08$ & $4.12 \mathrm{E}-09$ \\
\hline 4 & $1.80 \mathrm{E}-17$ & $3.28 \mathrm{E}-19$ & $7.43 \mathrm{E}-08$ & $1.35 \mathrm{E}-09$ & 4 & $1.24 \mathrm{E}-17$ & $9.76 \mathrm{E}-19$ & $5.11 \mathrm{E}-08$ & $4.03 \mathrm{E}-09$ \\
\hline 5 & $1.78 \mathrm{E}-17$ & $3.13 \mathrm{E}-19$ & $7.18 \mathrm{E}-08$ & $1.27 \mathrm{E}-09$ & 5 & $1.22 \mathrm{E}-17$ & $9.36 \mathrm{E}-19$ & $4.94 \mathrm{E}-08$ & $3.80 \mathrm{E}-09$ \\
\hline
\end{tabular}

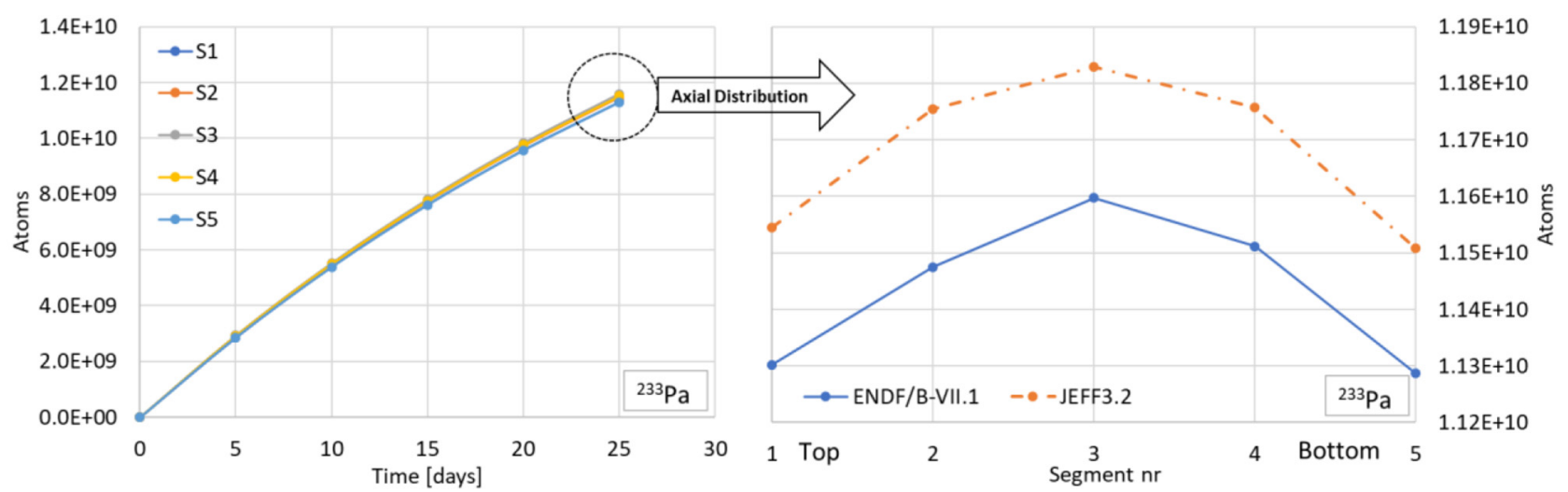

Fig. 6. Evolution of ${ }^{233} \mathrm{~Pa}$ during the irradiation time with final axial distribution in the sample.

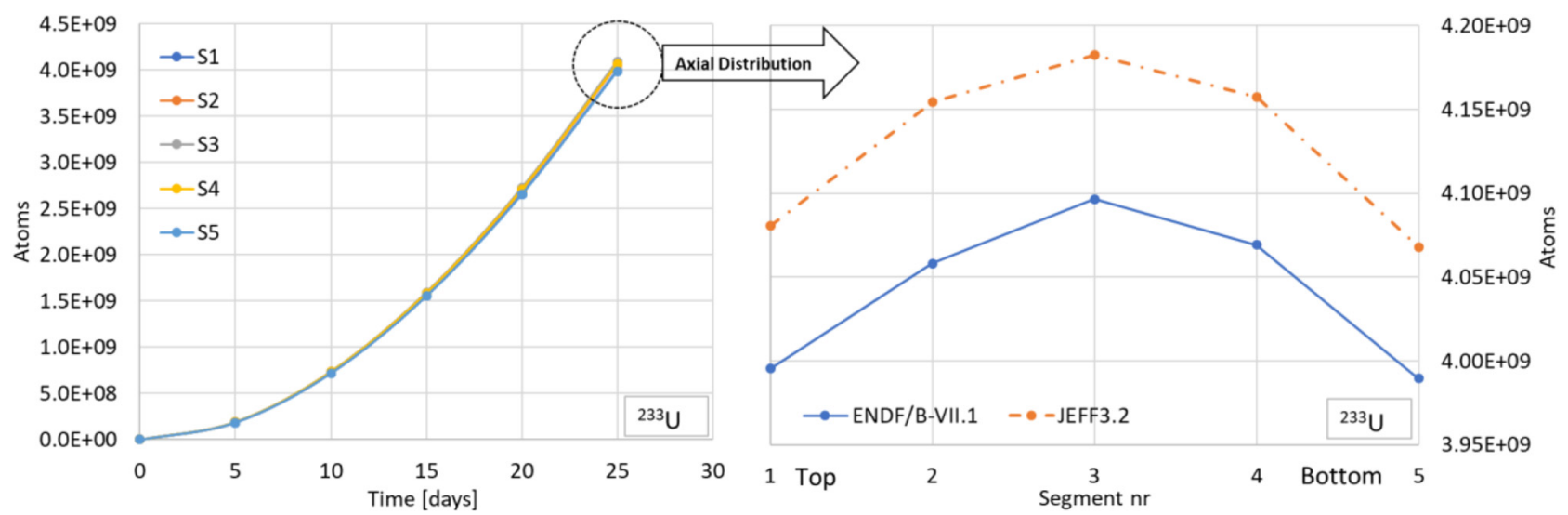

Fig. 7. Evolution of ${ }^{233} \mathrm{U}$ during the irradiation time with final axial distribution in the sample.

beginning of irradiation and for the formed isotopes $\left({ }^{233} \mathrm{~Pa}\right.$, $\left.{ }^{233} \mathrm{U},{ }^{239} \mathrm{~Np},{ }^{239} \mathrm{Pu}\right)$ - at the end of irradiation. The radiative capture and fission reaction rates for both nuclear data libraries are shown. The axial distributions of the reaction rates show a similar shape as the power and the number of distributions of atoms for the investigated isotopes, which proves the reliability of the calculations. The reaction rates were presented as possible input for other calculations considering similar nuclear set-ups for various numerical tools used by research teams. 


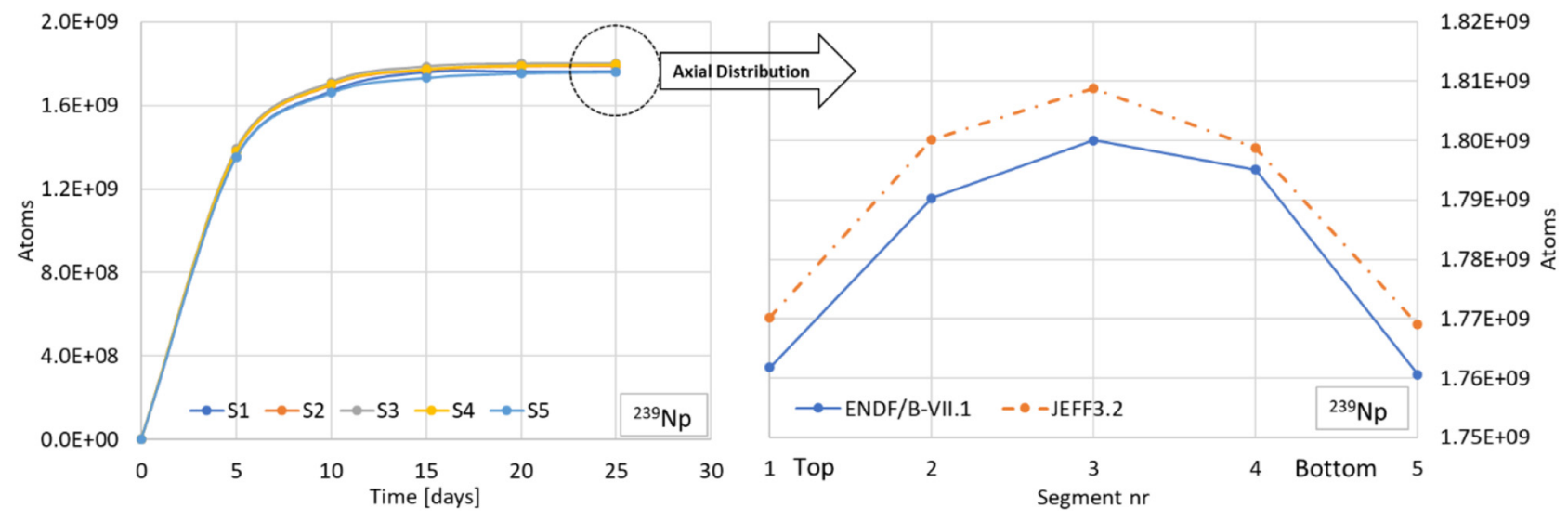

Fig. 8. Evolution of ${ }^{239} \mathrm{~Np}$ during the irradiation time with final axial distribution in the sample.

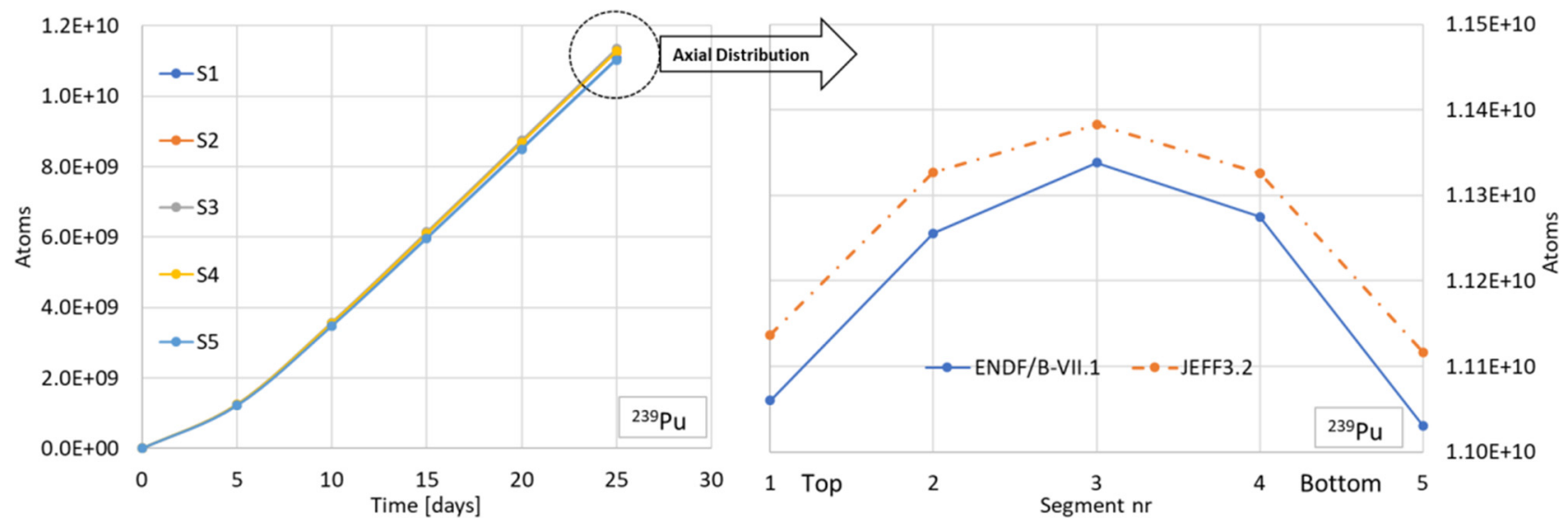

Fig. 9. Evolution of ${ }^{239} \mathrm{Pu}$ during the irradiation time with final axial distribution in the sample.

\subsection{Power}

Figures 10 and 11 present the power evolution during the sample irradiation and the final axial power distribution at the end of irradiation. In the $\mathrm{ThO}_{2}$ fuel sample, power increases smoothly from the beginning of irradiation due to the fissions on bred fissionable ${ }^{233} \mathrm{U}$, see Figure 7 . The power increase equals about $5 \%$ compared with the beginning of irradiation. The highest power for ENDF/BVII.1 libraries $\left(2.11 \times 10^{-9} \mathrm{~W}\right)$ was observed in the central segment of the fuel sample and the lowest $\left(1.85 \times 10^{-9} \mathrm{~W}\right)$ - in the top and bottom segments of the sample (1 and 5). The difference between the maximal and the minimal power for the central and the peripheral segments (1 vs. 3 ) during the whole irradiation time is about $7 \%$. The calculations with JEFF3.1. nuclear data libraries show power higher by about $3 \%$. The power level in the $\mathrm{UO}_{2}$ fuel sample is about one order of magnitude higher because of the initial presence of fissionable ${ }^{235} \mathrm{U}$ in natural uranium. Initially, power decreases due to the fuel depletion because of the fissions on ${ }^{235} \mathrm{U}$ and the lack of ${ }^{239} \mathrm{Pu}$. After a few days, power increases due to the formation of fissionable ${ }^{239} \mathrm{Pu}$ and the increased number of fissions. At the end of irradiation, power is about $1 \%$ higher. The maximal power in the central segment equals about $2.75 \times 10^{-8} \mathrm{~W}$ and the minimal power in the peripheral segments $-2.63 \times 10^{8} \mathrm{~W}$ for ENDF/B-VII.1 libraries, which gives a difference of about $5 \%$. In addition, the difference between the nuclear data libraries is much lower compared with the $\mathrm{ThO}_{2}$ fuel sample and equals about $0.3 \%$. The reason for the differences between the nuclear data libraries may be attributed to better knowledge on the isotope cross sections in the formation chain of ${ }^{239} \mathrm{Pu}$ than in the formation chain of ${ }^{233} \mathrm{U}$. The final axial power distribution corresponds to the neutron flux distribution in the samples and it is consistent with theoretical predictions, i.e. the closer to the neutron source, the higher the power is.

\subsection{Activity}

The activity in the sample segments for both fuel types is shown in Figures 12 and 13. The activity is higher in the case of the $\mathrm{UO}_{2}$ fuel sample due to the fissions on the residual ${ }^{235} \mathrm{U}$ and the formation of active fission products and due to the formation of minor actinides from the transmutations on ${ }^{238} \mathrm{U}$. The difference in the activity at the end of irradiation equals about $52 \%$ for each segment of the sample. The activity increases more rapidly after the initial jump in the case of $\mathrm{UO}_{2}$ and it reaches the equilibrium state faster at the end of irradiation. The 


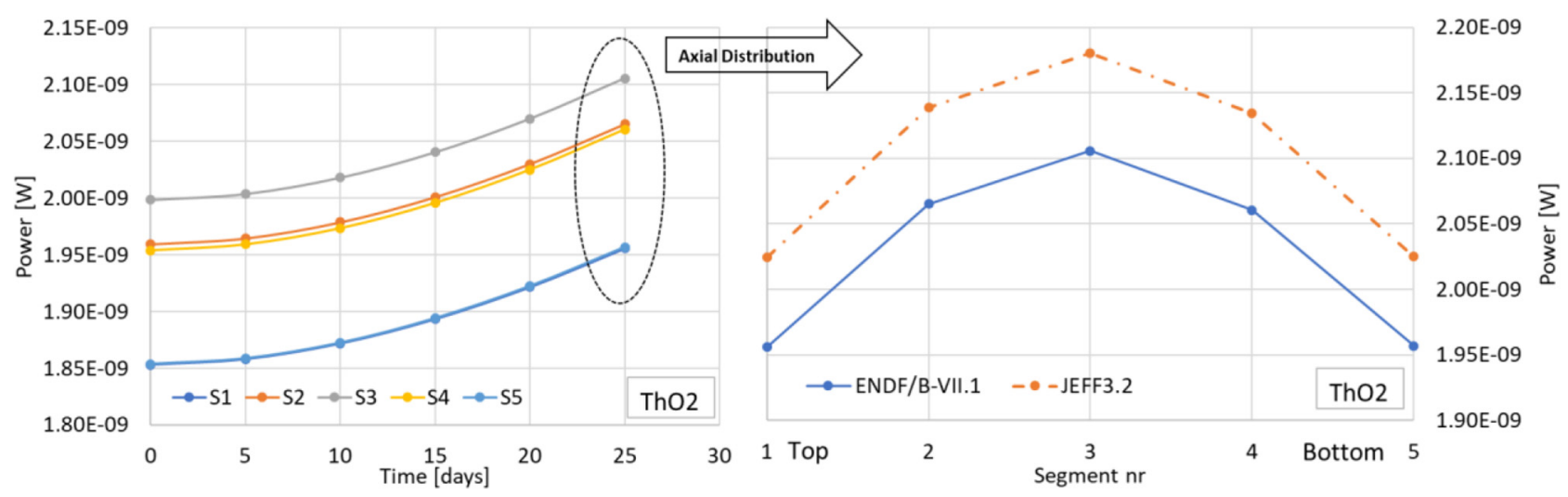

Fig. 10. Power evolution in the $\mathrm{ThO}_{2}$ sample during the irradiation time with final axial distribution.

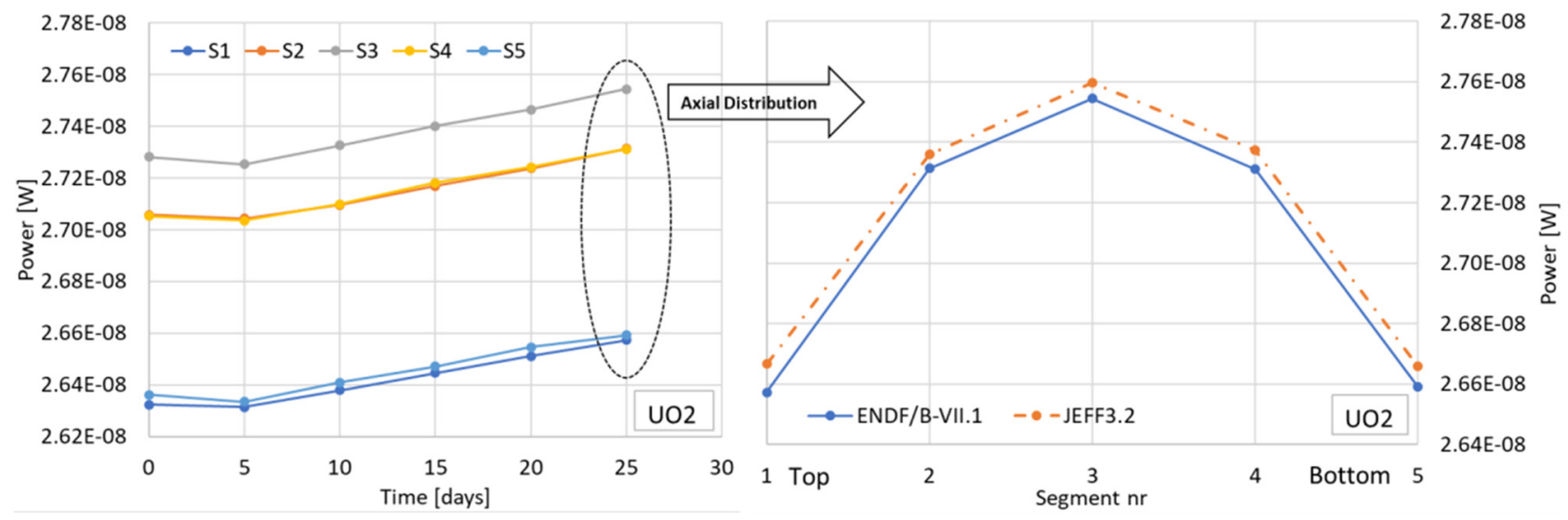

Fig. 11. Power evolution in the $\mathrm{UO}_{2}$ sample during the irradiation time with final axial distribution.

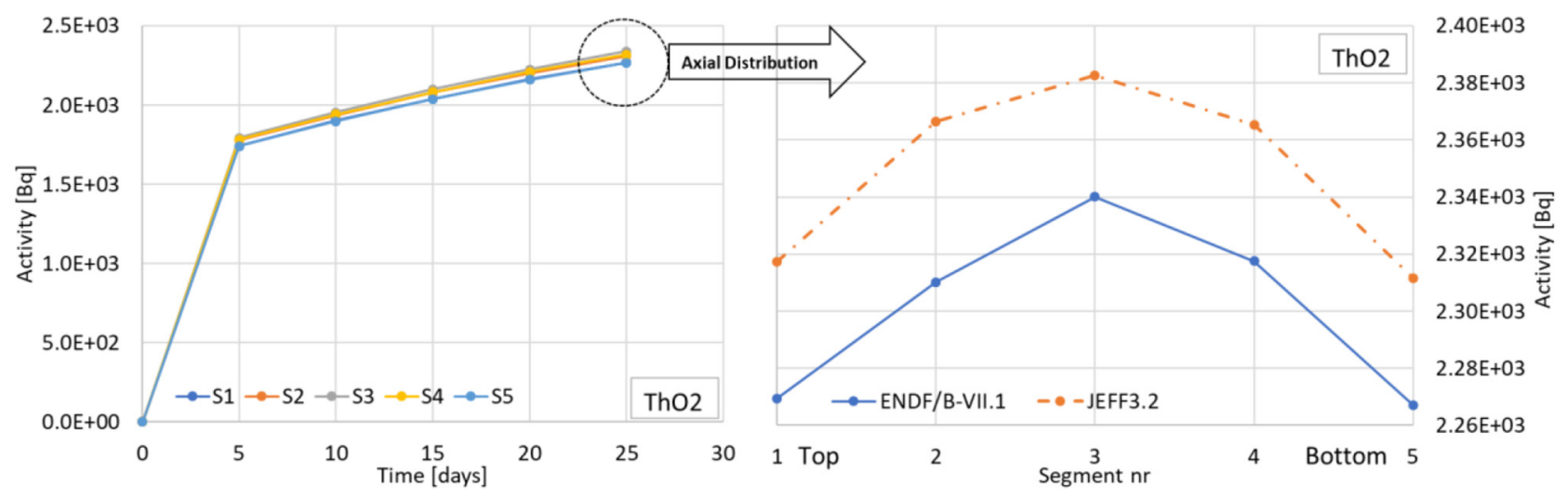

Fig. 12. Activity evolution in the $\mathrm{ThO}_{2}$ sample during the irradiation time with final axial distribution.

activity of $\mathrm{ThO}_{2}$ increases more slowly and during 25 days of irradiation it does not reach the equilibrium state because of the much longer time ( $\sim 10$ times) necessary for the formation of fissionable ${ }^{233} \mathrm{U}$ compared with the formation of ${ }^{239} \mathrm{Pu}$. Moreover, the transmutation and decay chain starting from ${ }^{232} \mathrm{Th}$ presents a longer path to the formation of notably radioactive minor actinides. The activity evolution and the final axial distribution is proportional to the power evolutions and distributions for both nuclear data libraries. Activity higher by about $2 \%$ was obtained for JEFF3.1 nuclear data libraries for the $\mathrm{ThO}_{2}$ fuel sample, while the difference for the $\mathrm{UO}_{2}$ sample equals about $0.4 \%$. The maximal activity for ENDF/BVII.1 libraries for the $\mathrm{ThO}_{2}$ fuel sample equals $2.34 \times 10^{3} \mathrm{~Bq}$, and for the $\mathrm{UO}_{2}$ fuel sample $-4.44 \times 10^{3} \mathrm{~Bq}$.

\section{Discussion and summary}

In the study, the design of irradiation experiments with a $\mathrm{ThO}_{2}$ and $\mathrm{UO}_{2}$ fuel sample was presented. The main 


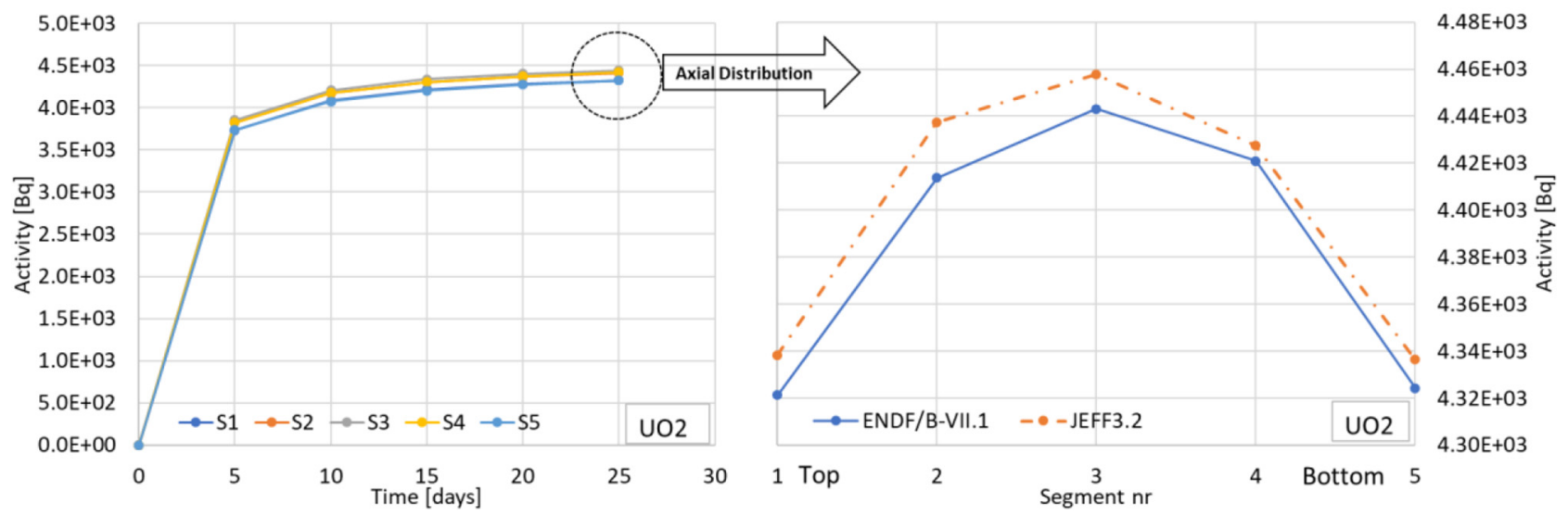

Fig. 13. Activity evolution in the $\mathrm{UO}_{2}$ sample during the irradiation time with final axial distribution.

dneutron characteristics of the sample located in the available experimental set-up were calculated using the developed numerical methodology based on the Monte Carlo modelling. The methodology includes the reconstruction of the experimental set-up using the functionalities of the MCB code and the high-performance computing capabilities for numerical calculations using the Prometheus supercomputer. The numerical simulations shows that some improvements are necessary for better planning of irradiation experiments. The irradiation time for the $\mathrm{ThO}_{2}$ sample should be longer in order to reach the equilibrium state of ${ }^{233} \mathrm{~Pa}$ and to cover full period of ${ }^{233} \mathrm{U}$ production. In the study, only the irradiation time with the neutron source embedded in the assembly is considered. For the experimental measurements, the decay of the remaining ${ }^{233} \mathrm{~Pa}$ and ${ }^{239} \mathrm{~Np}$ should be also considered to estimate the number of atoms of bred fissionable isotopes, especially ${ }^{233} \mathrm{U}$, after the source removal. The long-term activity of the samples should be also calculated for further technical and administrative maintenance of active fuel samples. Thus, the detailed analysis considering quantitative identification of the main isotopes contributing to the radiotoxicity of the spent Th fuel is recommended.

Moreover, the experimental measurements using the detectors available in the radiometric laboratory should be performed not immediately after the removal of the neutron source from the assembly but after the removal and opening of the fuel rod for the sample extraction and preparation for measurements. The basic requirement of the experiments (uncertainties of the measurement results) will be defined after choice of the measurement methodology. The time for technical maintenance of the sample should be included in the numerical modelling. The numerical modelling should also contain analysis of the gamma spectra in the experimental set-up, especially in the in lead-filtered media.

In the future experiments, the use of many configurations of the core is foreseen. Firstly, for the spectrum thermalisation, not only polyethylene but also graphite rods may be applied. Secondly, the assembly can be loaded with depleted or natural uranium fuel rods. Thirdly, the radio isotopic Cf neutron source could be changed to e.g. $\mathrm{Pu}-\mathrm{Be}$ source or even to D-T neutron generator. Finally, other irradiation positions of the sample are also possible, e.g. in the gap between the rods. The axial adjustment of the neutron source and the samples during long-term irradiations is also possible. The versatility of the assembly gives a perfect opportunity to perform many irradiation experiments for R\&D on the thorium and uranium fuel cycle in a different material and geometrical environment, e.g. for the development of IV Generation nuclear reactors [13]. The use of lead may help to enhance the knowledge about the fuel behaviour in Lead-Cooled Fast Reactor and Accelerator Driven Systems [7,14], while the use of graphite - in High Temperature Gas Reactors [15]. The current study has a preliminary character in order to demonstrate the capabilities of the available experimental set-up towards integral irradiation experiments and it focuses on the numerical approach. On the other hand, the experimental measurements would also help to validate the numerical methodology developed, especially the crosssection libraries, which show some differences, especially in the decay and transmutation chain to ${ }^{233} \mathrm{U}$.

The research was partially supported by PL Grid Infrastructure available at the Academic Computer Centre CYFRONET AGH. In addition, partial financial support of this study under the scientific subvention 16.16.210.476 by the Polish Ministry of Science and Higher Education is kindly acknowledged.

\section{References}

1. M. Oettingen, J. Cetnar, T. Mirowski, The MCB code for numerical modelling of fourth generation nuclear reactors, Comput. Sci. 16, 329-350 (2015)

2. M.B. Chadwick, M. Herman, P. Oblozinsky et al., ENDF/BVII.1 nuclear data for science and technology: cross sections, covariances, fission product yields and decay data, Nucl. Data Sheets, 112(12), 2887-2996 (2011)

3. A. Santamarina et al., The JEFF-3.1.1 nuclear data library, JEFF Report 22, Validation Results from JEF-2.2 to JEFF3.1.1, ISBN 978-92-64-99074-6 Nuclear Energy Agency, 2009

4. International Atomic Energy Agency, IAEA Nuclear Energy Series No. NF-T-2.4, Role of thorium to supplement fuel cycles of future nuclear energy systems, 2012

5. D.E. Serfontein, E.J. Mulder, Thorium-based fuel cycles: reassessment of fuel economics and proliferation risk, Nucl. Eng. Des. 271, 106-113 (2014) 
6. A. Nuttin, P. Guillemin, A. Bidaud, N. Capellan et al., Comparative analysis of high conversion achievable in thorium-fueled slightly modified CANDU and PWR reactors. Ann. Nucl. Energy 40, 171-189 (2011)

7. A. Wojciechowski, Criticality of the thorium burnup in equilibrium state, Prog. Nucl. Energy 92, 81-90 (2016)

8. A. Galahom, Minimization of the fission product waste by using thorium based fuel instead of uranium dioxide, Nucl. Eng. Des. 314, 165-172 (2017)

9. T. Kooyman, L. Buiron, Neutronic and fuel cycle comparison of uranium and thorium as matrix for minor actinides bearing-blankets, Ann. Nucl. Energy 92, 61-71 (2016)

10. S.I. Abdel-Khalik, P.A. Haldy, A. Kumar, Blanket design and calculated performance for the lotus fusion-fission hybrid experimental devices test facility, Fus. Sci. Technol. 5 (2), 189-208 (1984)
11. R.C. Martin, J.B. Knauer, P.A. Balo, Production, distribution and applications of californium-252 neutron sources, Appl. Radiat. Isotopes 53, 785-792 (2000)

12. J. Cetnar, General solution of Bateman equations for nuclear transmutations, Ann. Nucl. Energy 33, 640-645 (2006)

13. H. Gyorgy, Sz. Czifrus, The utilization of thorium in generation IV reactors, Progr. Nucl. Energy 93 (2016)

14. D.K. Mohapatra, S.S. Singh, A. Riyas, P. Mohanakrishnan, Physics aspects of metal fuelled fast reactors with thorium blanket, Nucl. Eng. Des. 265, 1232-1237 (2013)

15. I.V. Shamanin, V.M. Grachev, Y.B. Chertkov, S.V. Bedenko, O. Mendoza, V.V. Knyshev, Neutronic properties of hightemperature gas-cooled reactors with thorium fuel, Ann. Nucl. Energy 113, 286-293 (2018).

Cite this article as: Mikolaj Oettingen, Numerical design of thorium and uranium fuel samples irradiation in lead environment, EPJ Nuclear Sci. Technol. 6, 51 (2020) 